\title{
WILEY-VCH
}

DOI: $10.1002 /$ ((please add manuscript number))

Article type: Full Paper

\section{Investigation of Silver Nanoparticle Assembly Following Hybridisation with Different Lengths of DNA}

Tara Donnelly, Karen Faulds, and Duncan Graham*

T. J. Donnelly, Prof. K. Faulds, Prof. D. Graham

Centre for Molecular Nanometrology, WestCHEM, Department of Pure and Applied

Chemistry, University of Strathclyde, Technology and Innovation Centre, 99 George Street,

Glasgow, G1 1RD, UK

E-mail: Duncan.graham@strath.ac.uk

Keywords: nanoparticle, assembly, hybridization, DNA, silver

Assays involving the controlled assembly of oligonucleotide-functionalised nanoparticles have been widely investigated for the detection of short, specific sequences of DNA. The surface plasmon resonance changes that result from the near-field coupling of the nanoparticles provide a means for investigating formation of these assemblies. For these assays to be effective in practice, there needs to be rapid and efficient hybridisation of the functionalised nanoparticles with target DNA. However, it is known that the hybridisation rate is adversely affected by increased numbers of non-hybridising bases on the target DNA strand. This study investigates the DNA-directed assembly of oligonucleotide-functionalised silver nanoparticles, with the aim of identifying the parameters that will maximise hybridisation efficiency with long target sequences. The study shows that increasing the length of probes from 12 to 24 bases, and orientating them in a tail-to-tail rather than a head-to-tail configuration, results in significantly enhanced hybridisation with a long target sequence. The use of a volume excluding polymer such as dextran sulphate in the buffer also markedly improves hybridisation. This information will prove useful for researchers involved in the design of DNA-mediated nanoparticle assembly assays, particularly for the detection of long sequences of DNA. 


\section{WILEY-VCH}

\section{Introduction}

Nanoparticles, particularly silver and gold, have increasingly been used in the development of bio-detection assays for the identification of specific proteins or sequences of DNA. ${ }^{[1,2]}$ Detection of short, defined DNA sequences is important for diagnostic purposes, for instance to detect genetic mutations or to determine the cause of a bacterial, viral or fungal infection. These nanoparticle assays involve a change in the plasmonic properties of the functionalised nanoparticles that is triggered by the presence of a 'target' molecule, which can be measured spectroscopically. ${ }^{[3,4]}$ A common method for the detection of short DNA sequences is the use of a nanoparticle assembly assay, which incorporates two sets of nanoparticles, each of which is functionalised with a different, short oligonucleotide sequence that is complementary to a section of the target DNA. ${ }^{[3,4]}$ When the nanoparticle probes and target are mixed, sequence-specific hybridisation occurs, causing a controlled and directed aggregation of the nanoparticles, which results in a change in their surface plasmon resonance (SPR) frequency. This process was first described in 1996 by Mirkin et al., who investigated changes in the colour and extinction spectra of oligonucleotide-functionalised $13 \mathrm{~nm}$ gold nanoparticles in the presence of a synthetic target DNA sequence, ${ }^{[3]}$ and by Alivisatos et al., who described the DNA-directed assembly of $1.4 \mathrm{~nm}$ gold nanoparticles. ${ }^{[5]}$ Nanoparticle assembly assays have since been developed further to incorporate silver nanoparticles, ${ }^{[4]}$ magnetic nanoparticles, ${ }^{[6]}$ as well as mixed metal assays. ${ }^{[7]}$

A key factor when developing a successful assay is achieving rapid and efficient hybridisation between the functionalised nanoparticles and target DNA. A number of factors are known to affect the rate of this controlled aggregation, such as concentration 


\section{WILEY-VCH}

and length of the target sequence, ${ }^{[8]}$ hybridisation temperature and hybridisation buffer, ${ }^{[9,10]}$ and the position of the probe hybridisation region within target DNA. ${ }^{[11]}$ Methods have been developed to increase the hybridisation efficiency, such as the use of 'short internal complement' $\mathrm{DNA}^{[12]}$ and the use of spacers between the nanoparticle and probe DNA sequence to reduce steric hindrance between neighbouring oligonucleotide strands. ${ }^{[4,13-15]}$

The choice of hybridisation buffer is an important consideration in any molecular diagnostic assay, since the negative phosphate backbone on DNA requires charge screening between the probe and target oligonucleotides. Jin et al. found that the hybridisation rate was directly proportional to the salt concentration used in a gold nanoparticle sandwich assay, with no hybridisation taking place at sodium chloride concentrations less than $0.05 \mathrm{M} \cdot{ }^{[9]}$ Buffers containing polymers have also been used to improve the efficiency of DNA hybridisation, with dextran sulphate shown to accelerate the hybridisation between probe DNA and a 281 base pair (bp) target PCR fragment in a gold nanoparticle assembly assay. ${ }^{[10]}$ More recently, polyethylene glycol (PEG) was also shown to enhance DNA hybridisation to functionalised gold nanoparticles. ${ }^{[16]}$

During assay development, short lengths of synthetic target DNA are often used to assess the functioning and stability of the assay, with similar short lengths of probe DNA used to exactly match this, for instance two 12 -mer probes with a $24-$ mer target $^{[4,17]}$ or two 15-mer probes with a 30-mer target. ${ }^{[9]}$ However, for the detection of clinically relevant DNA, the target sequence is likely to be much longer than the combined length of the probe DNA sequences, which may have a significant effect on the formation of the nanoparticle assemblies. Smith et al. investigated the effects of changing the length of the linker DNA on the kinetics of assembly and the melting transitions of a gold 


\section{WILEY-VCH}

nanoparticle assembly assay. ${ }^{[8]}$ Two 12 -mer probe sequences were used throughout, while the target sequence was altered by adding non-hybridising bases either to the centre of the target DNA (i.e. as 'gaps') or to each end of the hybridising portion of the target DNA (i.e. as 'overhangs'). In both cases, the nanoparticle assembly process was found to be slower and the melting temperature decreased as more nucleotides were present, either as gaps or overhangs. ${ }^{[8]}$ The effects of increasing the length of linker DNA as a means of increasing the distance between the nanoparticles following the assembly process has also been investigated, establishing that the rate of nanoparticle assembly was reduced as the length of linker DNA was increased both for gold ${ }^{[17]}$ and silver ${ }^{[18]}$ nanoparticles. In each of these examples, the length of probe DNA has remained constant while the linker DNA length has increased by adding bases that are not involved in hybridisation with probe DNA. Here we assess the effects of both longer target and longer probe DNA sequences on the nanoparticle assembly process.

It is worth noting that this paper does not aim to accurately define the relationship between nanoparticle separation and hybridisation. This would not be possible from the data shown since there is known to be some flexibility in the DNA hybrids formed as a result of the breaks in the probe DNA, ${ }^{[3,19]}$ as well as flexibility from the hexaethylene glycol spacer units that are present between the nanoparticle and probe DNA sequence. Rather, it is intended to give practical advice for the design of DNA probes in nanoparticle assembly assays, particularly when used for the detection of long sequences of target DNA.

\section{Results and Discussion}

When two nanoparticles are brought into close proximity, coupling of the surface plasmons leads to a red-shift in the SPR peak. The extent of the shift is distance 


\section{WILEY-VCH}

dependent, with an approximately exponential decrease in the extent of the red-shift with increasing nanoparticle separation, and no interaction when the distance between the nanoparticles is greater than 2.5 times their diameter. ${ }^{[20,21]}$ For the DNA-directed assembly of functionalised silver nanoparticles (Figure 1a), changes in the formation of the nanoparticle assemblies under different assay conditions were measured by plotting the ratio of the extinction peak height of the red-shifted assemblies over that of the individual nanoparticles, i.e. extinction $530 /$ extinction $_{410}$ as a function of time following addition of the target DNA (see ESI Figure S1). While the size of the aggregate peak does change slightly for different probe and target lengths, it is very broad and does not vary significantly from a $530 \mathrm{~nm}$ maximum in any of the situations tested in these experiments, and therefore the change in extinction ratio reflects the change in $410 \mathrm{~nm}$ peak reduction for all of the results presented. Note that the schematic illustrated in Figure 1a is a simplified view of nanoparticle assembly in two dimensions, whereas in reality the assembly takes place in three dimensions resulting in a much less ordered assembly process and the production of a range of different sized clusters, as can be seen from the very broad red-shifted peak in Figure S1. ${ }^{[18]}$

\subsection{Effects of Probe Length and Orientation}

In order to determine the effects on the nanoparticle assembly process of varying the length and orientation of probe DNA, a range of oligonucleotide-functionalised silver nanoparticles were prepared using probe sequences of varying lengths. A thiol tether was attached to the $3^{\prime}$ or 5 ' end of the oligonucleotide sequence such that the nanoparticles were arranged in either a 'head-to-tail' or a 'tail-to-tail' orientation upon hybridisation with target DNA (Figure 1b). The number of bases on each probe was varied between 12 and 30, allowing ten different probe combinations to be compared; 3 in a head-to-tail and 7 in a tail-to-tail configuration (Figure 2). Full details of the probe 


\section{WILEY-VCH}

sequences used are contained in the Experimental section. In all cases, the combined probe sequence was exactly complementary to a central section of the target DNA, with no gaps.

Each combination of probes was first hybridised with a 144-base length of target DNA (Target $\left.{ }_{144}\right)$, using the same conditions throughout (10 $\mathrm{pM}$ of each probe, $1 \mathrm{nM}$ of target, $25^{\circ} \mathrm{C}, 3 \% \mathrm{w} / \mathrm{v}$ dextran sulphate in $0.3 \mathrm{M}$ PBS buffer). This target sequence was longer than the combined probe DNA length for all of the probe combinations investigated, as illustrated in Figure 1b. For the head-to-tail probe combinations, the extent and rate of nanoparticle assembly during hybridisation with Target ${ }_{144}$ was enhanced as the length of P1 DNA increased from 12 to 24 bases, as noted by a larger and more rapid change in the extinction profile (Figure 3a). Similarly, hybridisation efficiency was increased for the tail-to-tail combinations as the combined probe DNA length increased from 24 to 48 bases, with a reduction in hybridisation for the two 30-mer probes (Figure 3b). Duplicate blank samples (i.e. probes and buffer only) were also analysed for each probe combination; in all cases there was no significant change in the height or width of the silver nanoparticle plasmon throughout the analysis period, indicating that all probes were stable in the buffer used (data not shown). Comparisons can also be made for the $\mathrm{P} 1_{12}-\mathrm{P} 2_{12}, \mathrm{P} 1_{18}-\mathrm{P} 2_{12}$ and $\mathrm{P} 1_{24}-\mathrm{P} 2_{12}$ probe combinations in either a head-to-tail or tail-totail orientation when hybridised to Target ${ }_{144}$. In each case, the hybridisation rate was higher when the nanoparticles were spaced further apart in the tail-to-tail configuration (see ESI Figure S2), which is likely due to the reduced electrostatic and steric hindrance between the silver nanoparticles.

When the length of probe DNA is increased, a number of complex and interacting factors may be involved that could potentially affect hybridisation with a long target sequence, 


\section{WILEY-VCH}

including: increased base-pair interactions between probe DNA and the long target DNA, which would lead to stronger, more thermodynamically favourable duplexes compared to probes with fewer bases available for hybridisation; increased nanoparticle separation, which could result in reduced electrostatic and steric hindrance between the nanoparticles and reduced electrostatic and steric repulsion between the target overhangs and the nanoparticles as the effective length of the overhangs is reduced due to a longer hybridising section. Other minor contributory factors may include slower diffusion within the hybridising buffer of nanoparticles bound to longer probe sequences, possible secondary structure formation and greater strand-to-strand interactions for the longer probe DNAs, and reduced inter-particle salt concentrations due to smaller overlaps of the electric double layers surrounding the DNA-nanoparticle conjugates. ${ }^{[18]}$ A combination of some or all of these factors could potentially affect the nanoparticle assembly process with long target sequences.

When Storhoff et al. investigated the aggregation rate of DNA-gold nanoparticle conjugates in the presence of varying lengths of dsDNA linker, they found that longer linkers resulted in a slower rate of aggregation. ${ }^{[17]}$ Guerrini et al. also found that increasing the length of dsDNA linker between DNA-silver nanoparticle conjugates led to a reduction in the rate of hybridisation. ${ }^{[18]}$ However, in both cases the length of the probe DNA remained constant throughout, and therefore the number of possible basepair interactions in the hybridising duplexes was unchanged. In order to separate out the effects of changing probe DNA length with changing number of base pair interactions, as well as any electro-steric effects with target overhangs, the nanoparticle assembly process was assessed using a selection of probe length combinations hybridised with a 24-base length of target DNA. In this case, the length of duplex formed remained constant (24 bp), while the probe length and configuration was varied. Under these 


\section{WILEY-VCH}

conditions, the rate of hybridisation was found to decrease as the length of probe DNA was increased, and was also slower for the two 12-mer probes when in a tail-to-tail configuration compared with head-to-tail (Figure 4). For short target sequences, therefore, probe orientations that result in increased nanoparticle separation lead to a reduction in hybridisation, in agreement with the work of Storhoff et al. ${ }^{[17]}$ and Guerrini et al. ${ }^{[18]}$ This may be due to a combination of slower diffusion of the longer nanoparticleprobe conjugates, increased strand-strand interactions in longer probes, and the electrostatic repulsion between hybridising and non-hybridising bases that hinders formation of the duplex. ${ }^{[22]}$

Figure 3 and 4 show that the nanoparticle assembly process is enhanced for all conjugate combinations upon hybridisation with Target 24 compared with Target $_{144}$, noting also that the shorter target is present at a lower concentration $(500 \mathrm{pM}$ of Target 24 vs. $1 \mathrm{nM}$ of Target $\left.{ }_{144}\right)$. The length of target DNA is known to have an impact on the nanoparticle assembly process, with Smith et al. reporting that the presence of 'overhangs' in the target reduced the rate of hybridisation in the assembly of DNA-functionalised gold nanoparticles. ${ }^{[8]}$ Further evidence is provided for this silver nanoparticle assembly assay, whereby doubling the length of the target DNA from 24 to 48 bases was shown to significantly reduce the rate and extent of hybridisation with two 12-mer probes, and increasing the length further to 96 bases resulted in no obvious hybridisation (see ESI Figure S3). In all cases the target length was extended by an equal number of nucleotides on each side of the probe region such that the hybridising region remained in the centre (Figure S3a). The reduction in the rate of hybridisation with increasing target length is likely to be due to a combination of factors including the slower diffusion rate of the longer targets in the hybridisation buffer, ${ }^{[17]}$ steric hindrance from the long strands that may limit the extent of hybridisation possible between neighbouring nanoparticles, ${ }^{[17]}$ 


\section{WILEY-VCH}

electrostatic repulsion between the non-hybridising bases on the longer targets and probe DNA on the nanoparticles, ${ }^{[8]}$ and potential secondary structure formations for the long target sequences. The dramatic reduction in hybridisation efficiency with increasing target length could have significant implications for the use of nanoparticle assembly assays for detection of DNA in biological samples. ${ }^{[8]}$

\subsection{Effects of Buffer}

The results in Figure 3 and 4 used a 3\% w/v dextran sulphate in 0.3 M PBS buffer for hybridisation. Dextran sulphate was added to the buffer as this has been reported to increase the hybridisation rate of two DNA strands due to its volume excluding effect. ${ }^{[23]}$ This polymer was also used to successfully increase the rate of hybridisation of long target DNA with oligonucleotide-functionalised gold nanoparticles, ${ }^{[10]}$ however we are unaware of it being previously used in a silver nanoparticle assembly assay. By using increasing concentrations of dextran sulphate $(3 \%, 5 \%$ and $7 \% \mathrm{w} / \mathrm{v}$ all in $0.3 \mathrm{M} \mathrm{PBS})$, nanoparticle assembly was enhanced for the hybridisation of two 12-mer probes with Target $_{144}$ (see ESI, Figure S4). Note that no hybridisation was observed with $0 \%$ dextran sulphate (see ESI, Figure S3). However, some instability was observed for the blank sample in the $7 \%$ dextran buffer, indicating that there may be some non-specific hybridisation taking place between the probe DNA strands, or increased nanoparticle interaction as the nanoparticles are forced into closer proximity. Some instability was also observed for the longer probe sequences with the $5 \% \mathrm{w} / \mathrm{v}$ dextran sulphate buffer (data not shown), therefore 3\% dextran was chosen for use throughout.

Similar comparisons were also carried out using polyethylene glycol (PEG) 10,000 in place of dextran sulphate as this has also previously been shown to increase the kinetics of hybridisation in a gold nanoparticle assembly assay, ${ }^{[16]}$ although again we are unaware 


\section{WILEY-VCH}

of its use in a silver nanoparticle assembly assay. PEG 10,000 was shown to produce very similar results to the dextran sulphate, with the rate of hybridisation and probe instability increasing with increasing PEG concentration (see ESI, Figure S5). PEG 2,000 in 0.3 M PBS buffer was also investigated but gave a significantly reduced level

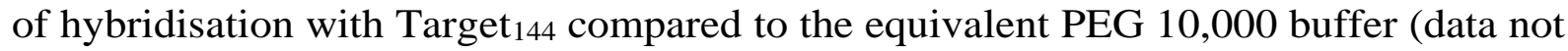
shown). Note that increasing the salt concentration of the buffer from $0.3 \mathrm{M}$ PBS to $1 \mathrm{M}$ PBS (i.e. $10 \mathrm{mM}$ phosphate buffer with either $0.3 \mathrm{M}$ or $1 \mathrm{M} \mathrm{NaCl}$ ) did not significantly improve the hybridisation between Target 144 and two 12-mer probes (see ESI, Figure S6).

\subsection{Melting Transitions}

The melting temperature $\left(\mathrm{T}_{\mathrm{m}}\right)$ of DNA-nanoparticle assemblies, i.e. the temperature at which the probe and target DNA strands dissociate, indicates the thermodynamic stability of the system. The sharp, reproducible melting transitions can be important to allow detection of single base mismatches in both gold $^{[9]}$ and silver nanoparticle assemblies. ${ }^{[4]}$ A number of factors are known to affect the $T_{m}$ of a nanoparticle assembly assay, such as probe and target concentration, ${ }^{[13]}$ probe density, particle size, salt concentration, length of spacer, and probe orientation. ${ }^{[9]}$

The $\mathrm{T}_{\mathrm{m}}$ was determined in this current assay by measuring the change in intensity of the SPR peak at $410 \mathrm{~nm}$ with temperature; this change occurs due to the nanoparticle assemblies separating and reverting back to free nanoparticle conjugates in suspension as the probe and target DNA strands dissociate once the melting temperature is reached. $410 \mathrm{~nm}$ was chosen rather than $530 \mathrm{~nm}$ as the changes in peak intensity are much more distinct at this lower wavelength, particularly for situations where there are very low levels of aggregation, allowing a more accurate measurement of the melting temperature. 


\section{WILEY-VCH}

Measurements for a range of probes hybridised with Target ${ }_{144}$ showed that an increase in probe length led to an increase in $\mathrm{T}_{\mathrm{m}}$ for both the head-to-tail and tail-to-tail combinations (see Table 1 and ESI, Figure S7). This change is significant, with a $\geq$ $30{ }^{\circ} \mathrm{C}$ increase in $\mathrm{T}_{\mathrm{m}}$ for the two 30-mer probes compared to two 12-mer probes in a tailto-tail orientation. This is likely due to the increased base stacking interactions ${ }^{[8]}$ and cooperative melting effects due to the longer duplexes formed, as well as a possible increase in the amount of duplex formed ${ }^{[13]}$ due to increased hybridisation, as explained in the previous section. It should be noted that the target concentration and hybridisation period prior to heating were both greater than for the kinetics experiments, which was required to reduce the effect of varying quantity of duplex formed between probe combinations.

To determine the extent of the effect of the increased duplex length on $\mathrm{T}_{\mathrm{m}}$, the two 30 mer probes (tail-to-tail orientation) were also hybridised with Target 24 under the same conditions. In this case the $\mathrm{T}_{\mathrm{m}}$ dropped to $62.5 \pm 0.2{ }^{\circ} \mathrm{C}$, compared with $>88^{\circ} \mathrm{C}$ when hybridised with Target ${ }_{144}$ (Table 1). This result is interesting since Smith et al. found that there was a large decrease in $T_{m}$ as a result of increased 'overhangs' in the target DNA due to electrostatic repulsion, ${ }^{[8]}$ and therefore highlights that the formation of stronger duplexes as a result of more probe/target DNA interactions is more significant than the reduced electrostatic repulsion achieved by moving to a shorter target length. Comparison of the $\mathrm{T}_{\mathrm{m}}$ values for the same lengths of duplex with a change in probe orientation reveals that a tail-to-tail orientation consistently gives rise to a higher melting temperature than head-to-tail (Table 1), similar to the result observed by previous authors investigating a gold ${ }^{[9]}$ and a silver ${ }^{[4]}$ nanoparticle assembly assay. This effect is likely due to the reduced steric hindrance and electrostatic repulsion as the nanoparticles 


\section{WILEY-VCH}

are spaced further apart, combined with the increased hybridisation efficiency, as found in the hybridisation experiments. ${ }^{[9]}$

\section{Conclusions}

Increasing the length of target DNA was shown to limit the nanoparticle assembly process for silver nanoparticles functionalised with short oligonucleotide sequences. The effect was more pronounced with increasing target length, and is thought to be primarily due to electrostatic and steric hindrance between the non-hybridising nucleotides on the target sequence and the nanoparticle-probe conjugates, along with slower diffusion of the longer target sequences. The use of a volume excluding polymer, such as dextran sulphate or polyethylene glycol, was shown to dramatically improve the hybridisation of short probes with long target sequences, due to the probes and target being forced into closer proximity by the polymer. To ensure stability of a range of different nanoparticle conjugates, a buffer consisting of $3 \% \mathrm{w} / \mathrm{v}$ dextran sulphate in $0.3 \mathrm{M}$ PBS was used for most of this work. For target DNA that is longer than the combined probe DNA, increasing the length of probe DNA and using a tail-to-tail configuration was shown to significantly enhance hybridisation. This is believed to be due to the creation of by ereating more base-pair interactions and reduction of electrostatic and steric interactions within the system, and leads to the formation of very strong duplexes with high melting temperatures. However, if the length of probe DNA is increased too much, hybridisation rate is reduced due to slower diffusion of the conjugates and potential strand-strand interactions and secondary structure effects in the probe DNA. For short target sequences, maximum hybridisation was achieved when the combined probe DNA length matched the length of the target DNA, highlighting the range of complex interactions that take place in a nanoparticle assembly assay. 


\section{WILEY-VCH}

\section{Experimental Section}

Oligonucleotide Probe and Target Sequences: All oligonucleotide sequences were purchased from AtdBio (Southampton). Probe sequences were treated with DTT and purified by HPLC before use, target sequences were used without further purification. Probe sequences are detailed below ( 5 ' to $3^{\prime}$ direction) where (HEG) $)_{3}$ denotes three hexaethylene glycol units, used as a spacer between the nanoparticle and the probe sequence:

$$
\begin{aligned}
& \text { 5'-P1 } 1_{12}: \quad \text { SH-(HEG) } 3 \text {-TCA AAG TAA TCG } \\
& \text { 3'-P2 12: TCC TGG TTC GCC-(HEG) }{ }_{3}-\mathrm{SH} \\
& \text { 5'-P212: } \quad \text { SH-(HEG) } 3 \text {-TCC TGG TTC GCC } \\
& \text { 5'-P1 } \left.1_{18}: \quad \text { SH-(HEG) }\right)_{3} \text {-ATT TCC TCA AAG TAA TCG } \\
& \text { 3'-P2 } \left.{ }_{18} \text { : TCC TGG TTC GCC CGA GGC-(HEG) }\right)_{3}-\mathrm{SH} \\
& \text { 5'-P1 }{ }_{24}: \quad \text { SH-(HEG) } \quad \text {-ACT CTA ATT TCC TCA AAG TAA TCG } \\
& \text { 3'-P224: TCC TGG TTC GCC CGA GGC TAG CCA-(HEG) }{ }_{3}-\mathrm{SH} \\
& \text { 5'-P1 } 130 \text { SH-(HEG) } \quad \text {-TTG AAC ACT CTA ATT TCC TCA AAG TAA TCG } \\
& \text { 3'-P230: TCC TGG TTC GCC CGA GGC TAG CCA GAA GGA-(HEG)3-SH }
\end{aligned}
$$

Target sequences were as follows ( $5^{\prime}$ to $\left.3^{\prime}\right)$ :

Target $_{24}: \quad$ GGC GAA CCA GGA CGA TTA CTT TGA

Target 48 TGG CTA GCC TCG GGC GAA CCA GGA CGA TTA CTT TGA GGA AAT TAG AGT

Target 96: CTG TTG CGG CCG GGT CTT TCC TTC TGG CTA GCC TCG GGC GAA CCA GGA CGA TTA CTT TGA GGA AAT TAG AGT GTT CAA AGC AGG CCT TTG CTC GGA

Target $_{144}$ CGG ACG GTC TAC CTA TGG TAA GCA CTG TTG CGG CCG GGT CTT TCC TTC TGG CTA GCC TCG GGC GAA CCA GGA CGA TTA CTT TGA 


\section{WILEY-VCH}

GGA AAT TAG AGT GTT CAA AGC AGG CCT TTG CTC GGA TAT ATT AGC ATG GAA TAA TAG AAT

Buffer Preparation: $60 \mathrm{mM}$ phosphate buffer prepared by adding $60 \mathrm{mM}$ aqueous sodium di-hydrogen phosphate (Fluka) to $100 \mathrm{~mL}$ of $60 \mathrm{mM}$ aqueous di-sodium hydrogen phosphate (Fluka) until pH 7.3 reached. Phosphate buffered saline (PBS, 0.3 M, pH 7) prepared by combining $60 \mathrm{mM}$ phosphate buffer with $2 \mathrm{M} \mathrm{NaCl}$ to give a solution containing $10 \mathrm{mM}$ phosphate and $0.3 \mathrm{M} \mathrm{NaCl}$. Dextran sulfate sodium salt from Leuconostoc spp. (average MW > 500,000) provided by Sigma-Aldrich. Polyethylene glycol (PEG) 10,000 provided by Fluka, PEG 2,000 provided by Alfa Aesar. Dextran sulphate and PEG buffers prepared by combining a mass of polymer to a volume of 0.3 M PBS to achieve the required percentage weight of polymer per unit volume of PBS; buffers stored in a fridge and prepared fresh weekly.

Reaction Kinetics Experiments: A Varian Cary 300Bio UV-Visible spectrophotometer with a temperature controller attachment was used for all extinction measurements. Experiments were carried out by combining probe 1 and probe 2 (10 pM of each probe) with a certain concentration of target (e.g. $1 \mathrm{nM}$ ) and buffer to a total volume of $800 \mu \mathrm{L}$ in a quartz cuvette. Spectra were collected every 10 min over a 90 min period, starting immediately after target addition, and samples were maintained at $25^{\circ} \mathrm{C}$ throughout. Each experiment was carried out in duplicate under the same conditions; all graphs show the average of these two samples. All spectra were converted to Excel for data analysis.

$T_{m}$ Measurements: Samples were prepared in the same way as for the reaction kinetics experiments and were left to hybridise for $3 \mathrm{hr}$ at $25^{\circ} \mathrm{C}$ before analysis. Melting transition measurements were then carried out, and involved heating the samples from 


\section{WILEY-VCH}

$25-70{ }^{\circ} \mathrm{C}$ (or $25-90{ }^{\circ} \mathrm{C}$ for samples with higher melting temperatures) at $1{ }^{\circ} \mathrm{C} / \mathrm{min}$ while monitoring the extinction at $410 \mathrm{~nm}$.

Silver Nanoparticle Synthesis: ${ }^{[24]} 89 \mathrm{~mL}$ of water was added to a conical flask along with freshly prepared aqueous sodium hydroxide (12.0 $\mathrm{mg}$ in $1 \mathrm{~mL}$ water, Sigma-Aldrich) and hydroxylamine hydrochloride (10.7 mg, Sigma-Aldrich). $\mathrm{AgNO}_{3}(17.0 \mathrm{mg}$ in $10 \mathrm{~mL}$ water, Aldrich) was added dropwise, with vigorous stirring of the solution throughout. The nanoparticles were used without further purification. All glassware was soaked in aqua regia for at least $2 \mathrm{hr}$ and thoroughly rinsed prior to nanoparticle synthesis. Diameter of synthesised silver nanoparticles was measured as $48 \pm 10 \mathrm{~nm}$, based on SEM image and using ImageJ software (see ESI Figure S8).

Conjugation of Silver Nanoparticles with Probe DNA: Silver nanoparticle-DNA conjugates were typically made according to the procedure developed by Zhang et al. ${ }^{[25]}$ : hydroxylamine-reduced silver nanoparticles $(321 \mathrm{pM}, 1 \mathrm{~mL})$ and probe oligonucleotide sequence (e.g. $21.6 \mu \mathrm{M}, 44.6 \mu \mathrm{L}, 3000: 1$ ratio) were added to an Eppendorf tube, followed by 2 aliquots of $500 \mathrm{mM}$ citrate. $\mathrm{HCl}$ buffer $(20 \mu \mathrm{L}$ each, $10 \mathrm{~min}$ gap between additions). Samples were left for $40 \mathrm{~min}$ then HEPES buffer (500 mM, $60 \mu \mathrm{L})$ and $\mathrm{NaCl}$ ( $2 \mathrm{M}, 200 \mu \mathrm{L}$ ) were added, the samples were then left for a further $45 \mathrm{~min}$ then centrifuged and resuspended in phosphate buffer $(10 \mathrm{mM}, 0.5 \mathrm{~mL})$. Centrifugation and resuspension was repeated twice more and samples were stored in a fridge until ready for use. The concentration of silver nanoparticles and conjugates was determined based on extinction measurements, using a molar extinction coefficient of $2.87 \times 10^{10} \mathrm{M}^{-1} \mathrm{~cm}^{-}$ $1[26]$ 


\section{WILEY-VCH}

Calculation of Number of Oligonucleotides per Nanoparticle: ${ }^{[27]}$ A fluorescent dye-labelled thiol-modified 12-base probe sequence [HS-Cy3-(HEG) 3 -TCTCAACTCGTA] was conjugated to hydroxylamine-reduced AgNPs following the standard procedure, and the conjugate concentration determined by extinction spectroscopy. A $100 \mu \mathrm{L}$ aliquot of conjugate was added to $100 \mu \mathrm{L}$ dithiothreitol (DTT, 1.0 M) in phosphate buffer ( $\mathrm{pH} 8.0,0.18 \mathrm{M}$ ) and left on a shaker overnight. Three separate samples were prepared in the same way to ensure accuracy in the calculated value. By next morning, all of the AgNPs had aggregated and dropped to the bottom of the Eppendorf tubes, indicating that the oligonucleotide probes had become detached from the nanoparticle surface. The samples were centrifuged $(6,000 \mathrm{rpm}, 20 \mathrm{~min})$ and the supernatants transferred to fresh vials; fluorescence measurements were carried out on aliquots of these supernatant solutions, in triplicate. Results were compared with control samples, prepared from a range of concentrations of probe sequence in phosphate buffer $(10 \mathrm{mM}, 80 \mu \mathrm{L})$, further diluted in a 1:1 ratio with 1.0 M DTT in $0.18 \mathrm{M}$ phosphate buffer prior to fluorescence measurements, such that the sample and control matrices were equivalent. The measured fluorescence level from the detached probe samples was then compared against a plot of fluorescence signal vs. quantity of probe sequence for the control samples and used to determine the quantity of probe sequence in the sample aliquots, which was then related to the number of DNA probes per individual silver nanoparticle based on the measured concentration of nanoparticle-probe conjugate. The number of oligonucletide strands per $48 \mathrm{~nm}$ silver nanoaprticle was calculated as approximately $1,300 \pm 200$, which compares well with the value calculated by Hurst et al. of 1,200 DNA strands per $50 \mathrm{~nm}$ AuNP. ${ }^{[27]}$

SEM Measurement: Scanning electron microscope (SEM) images were obtained using a FEI Sirion 200 ultra-high resolution Schottky field emission scanning electron microscope with FEI software. The SEM samples were prepared on silicon wafers as follows: the wafers were first washed with a methanol-soaked swab and placed in an oxygen plasma cleaner for 90 seconds. A positive surface charge was then created by applying a few drops of a solution of poly (diallyldimethylammonium chloride) (PDDA, $10 \mu \mathrm{L}$, Sigma Aldrich) in sodium chloride $(1 \mathrm{~mL}, 1 \mathrm{mM})$ to cover the wafer surface for 20 min, before rinsing with water and drying under a stream of nitrogen. The wafer was 


\section{WILEY-VCH}

then placed overnight in an Eppendorf tube containing $50 \mathrm{pM}$ of AgNP-oligonucleotide conjugate then rinsed with water and dried under a stream of nitrogen.

\section{Supporting Information}

Supporting Information is available from the Wiley Online Library or from the author. Data available: further comparisons of extinction spectra changes for different probe combinations, target lengths and buffers; melting transition spectra; SEM of AgNPs.

\section{Acknowledgements}

The authors wish to thank Renishaw Diagnostics Ltd. and the Engineering and Physical Sciences Research Council for funding of this work, and Dr. Samuel Mabbott for provision of the SEM image.

Received: ((will be filled in by the editorial staff))

Revised: ((will be filled in by the editorial staff)) Published online: ((will be filled in by the editorial staff))

[1] H. I. Peng, B. L. Miller, Analyst 2011, 136, 436.

[2] C. S. Thaxton, N. L. Rosi, C. A. Mirkin, MRS Bull. 2005, 30, 376.

[3] C. A. Mirkin, R. L. Letsinger, R. C. Mucic, J. J. Storhoff, Nature 1996, 382, 607.

[4] D. Thompson, A. Enright, K. Faulds, W. Smith, D. Graham, Anal. Chem. 2008, 80, 2805.

[5] A. Alivisatos, K. Johnsson, X. Peng, T. Wilson, C. Loweth, M. Bruchez, P. Schultz, Nature 1996, 382, 609.

[6] S. I. Stoeva, F. W. Huo, J. S. Lee, C. A. Mirkin, J. Am. Chem. Soc. 2005, 127, 15362.

[7] D. G. Thompson, R. J. Stokes, R. W. Martin, R. J. Lundahl, K. Faulds, D. Graham, Small 2008, 4, 1054.

[8] B. D. Smith, N. Dave, P.-J. J. Huang, J. Liu, J. Phys. Chem. C 2011, 115, 7851.

[9] R. Jin, G. Wu, Z. Li, C. Mirkin, G. Schatz, J. Am. Chem. Soc. 2003, 125, 1643. 


\section{WILEY-VCH}

[10] J. J. Storhoff, A. D. Lucas, V. Garimella, Y. P. Bao, U. R. Muller, Nat. Biotechnol. 2004, 22,883 .

[11] Y. Takeda, T. Kondow, F. Mafune, J. Phys. Chem. C 2008, 112, 89.

[12] A. E. Prigodich, O.-S. Lee, W. L. Daniel, D. S. Seferos, G. C. Schatz, C. A. Mirkin, J. Am. Chem. Soc. 2010, 132, 16296.

[13] A. K. R. Lytton-Jean, C. A. Mirkin, J. Am. Chem. Soc. 2005, 127, 12754.

[14] L. Barrett, J. Dougan, K. Faulds, D. Graham, Nanoscale 2011, 3, 3221.

[15] L. M. Demers, C. A. Mirkin, R. C. Mucic, R. A. Reynolds, R. L. Letsinger, R. Elghanian, G. Viswanadham, Anal. Chem. 2000, 72, 5535.

[16] X. Zhang, P.-J. J. Huang, M. R. Servos, J. Liu, Langmuir 2012, 28, 14330.

[17] J. Storhoff, A. Lazarides, R. Mucic, C. Mirkin, R. Letsinger, G. Schatz, J. Am. Chem. Soc. 2000, 122, 4640.

[18] L. Guerrini, F. McKenzie, A. W. Wark, K. Faulds, D. Graham, Chem. Sci. 2012, 3, 2262.

[19] C. J. Loweth, W. B. Caldwell, X. G. Peng, A. P. Alivisatos, P. G. Schultz, Angew. Chem., Int. Ed. 1999, 38, 1808.

[20] B. M. Reinhard, M. Siu, H. Agarwal, A. P. Alivisatos, J. Liphardt, Nano Lett. 2005, 5, 2246.

[21] K. H. Su, Q. H. Wei, X. Zhang, J. J. Mock, D. R. Smith, S. Schultz, Nano Lett. 2003, 3, 1087.

[22] L. Di Michele, B. M. Mognetti, T. Yanagishima, P. Varilly, Z. Ruff, D. Frenkel, E. Eiser, J. Am. Chem. Soc. 2014, 136, 6538.

[23] J. G. Wetmur, Biopolymers 1975, 14, 2517.

[24] N. Leopold, B. Lendl, J. Phys. Chem. B 2003, 107, 5723.

[25] X. Zhang, M. R. Servos, J. Liu, Chem. Commun. 2012, 48, 10114.

[26] J. Yguerabide, E. E. Yguerabide, Anal. Biochem. 1998, 262, 137. 
[27] S. J. Hurst, A. K. R. Lytton-Jean, C. A. Mirkin, Anal. Chem. 2006, 78, 8313.

a)

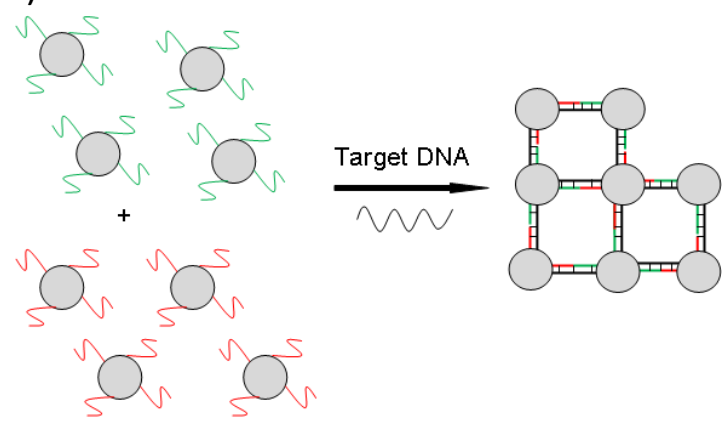

b)

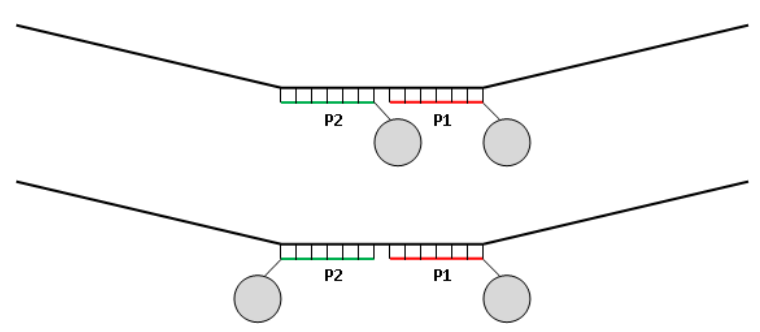

Figure 1. a) Simplified schematic of DNA-directed silver nanoparticle assembly in two dimensions; b) Schematic illustration of probe DNA (probe 1 in red, probe 2 in green) hybridising to a section of long target DNA (black). Each probe DNA sequence varies in length from 12 to 30 bases, and orientation upon hybridisation with target is either head-to-tail (top) or tail-to-tail (bottom).

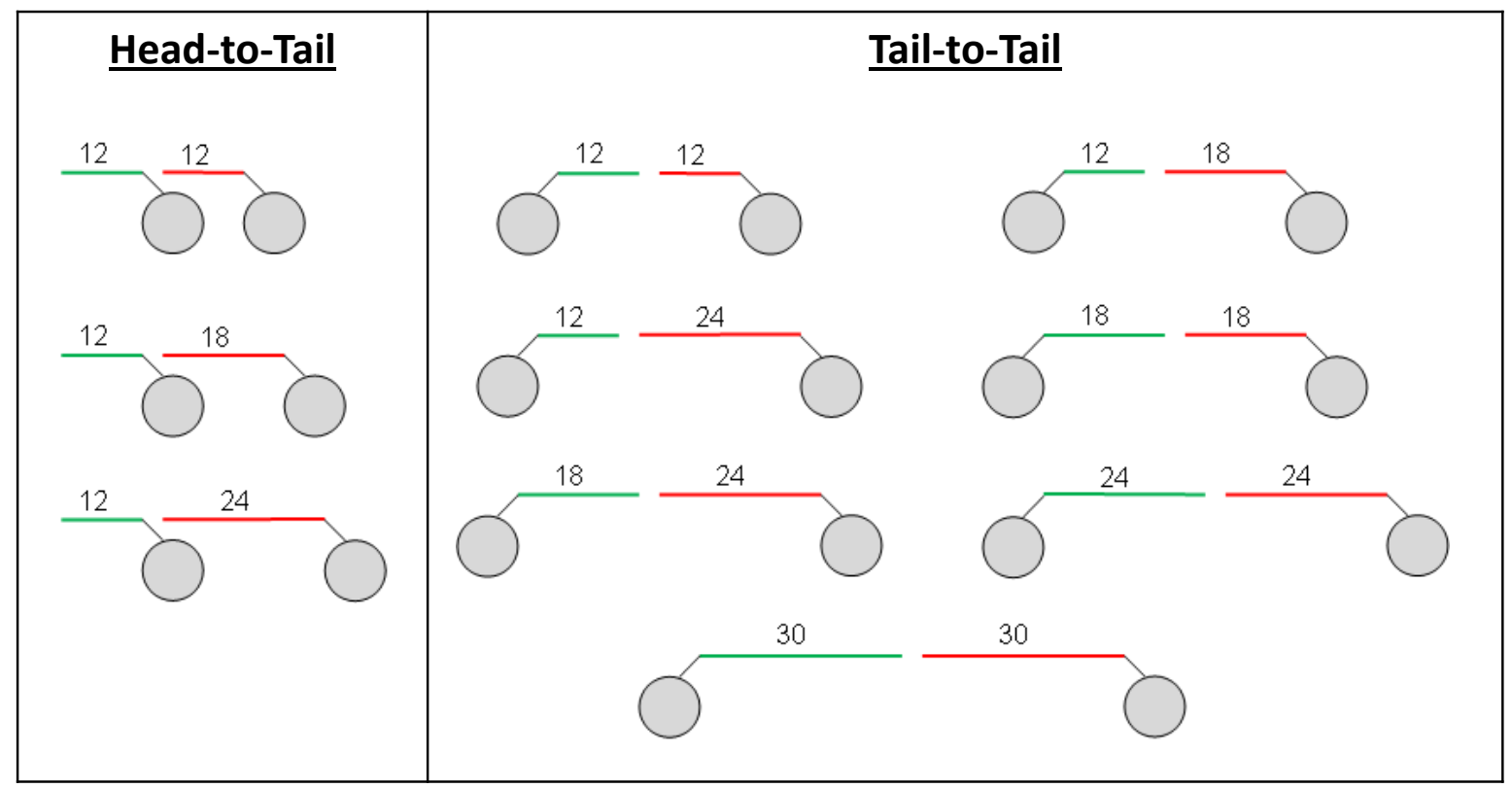

Figure 2. Schematic showing various combinations of different lengths of nanoparticle-bound probe DNA in head-to-tail and tail-to-tail orientations. P1 DNA is shown in red; P2 DNA is shown in green. All probe sequences are shown in a 3' to 5' direction. 

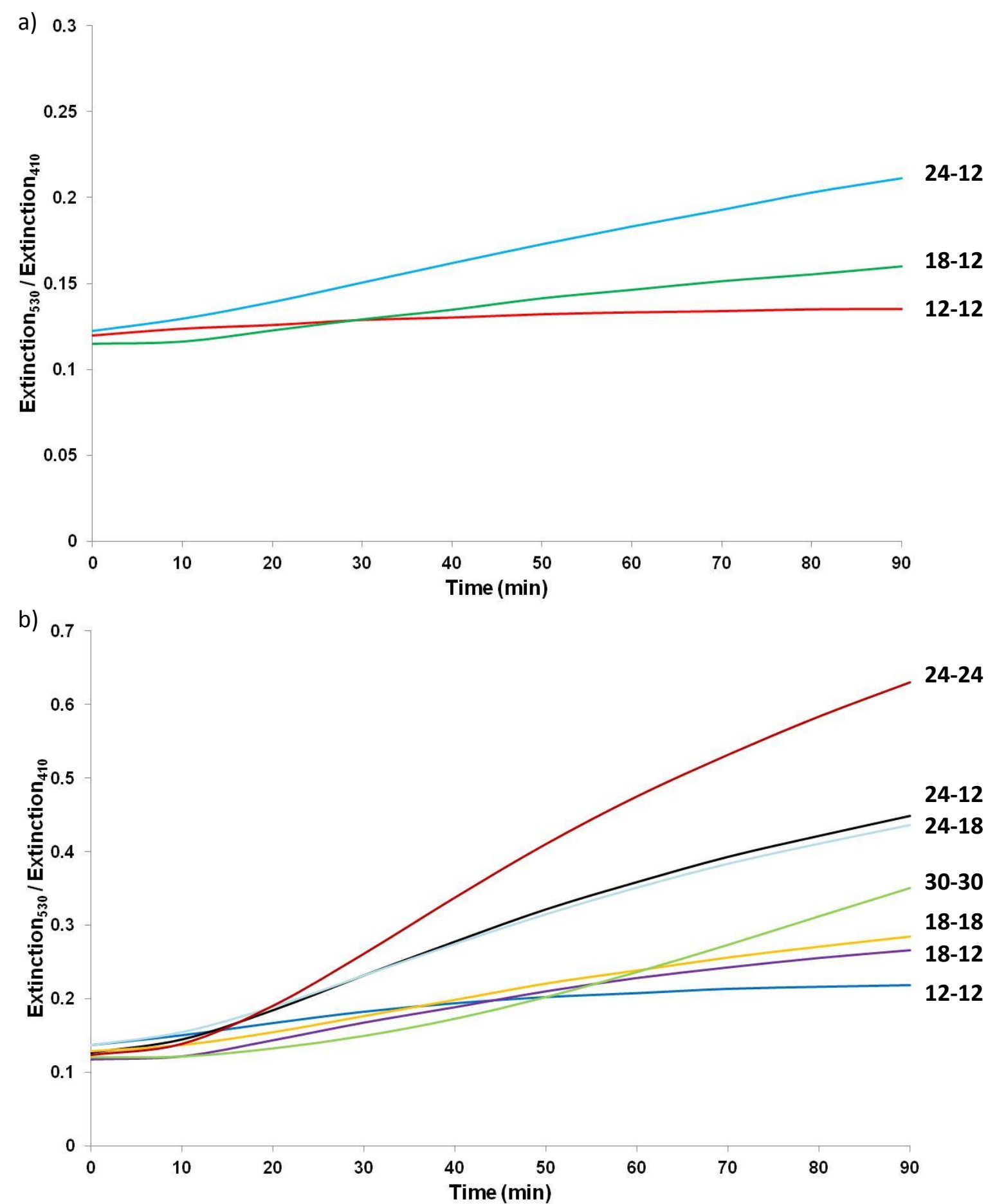

Figure 3. Changes in aggregation over a $90 \mathrm{~min}$ period following addition of $1 \mathrm{nM}$ of 144 base length target DNA to a solution of 10 pM P1 + 10 pM P2 in 3\% dextran sulphate/0.3 M PBS buffer for different a) head-to-tail and; b) tail-to-tail probe combinations. Captions to right of graphs indicate the number of bases in P1 and P2, respectively. 


\section{WILEY-VCH}

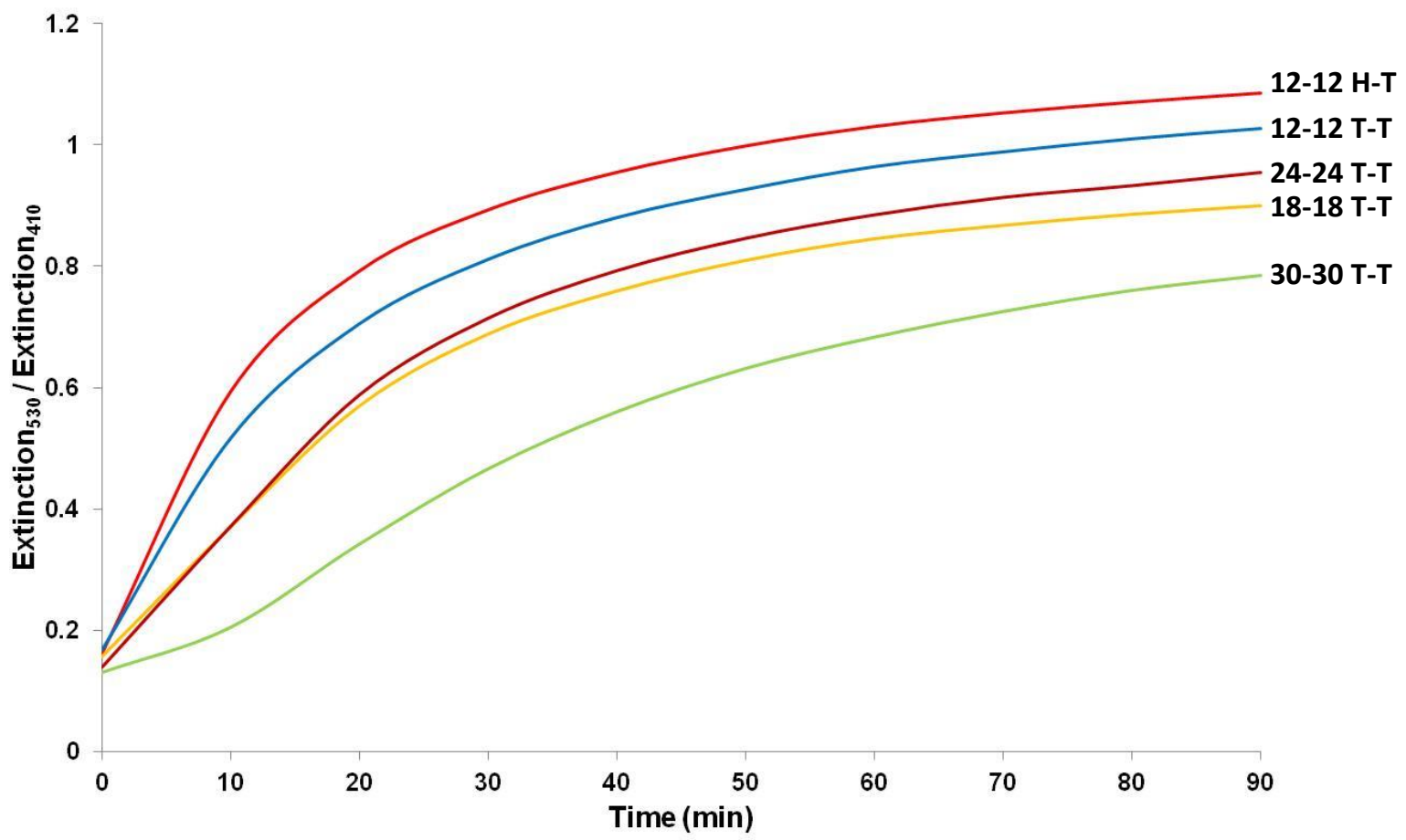

Figure 4. Changes in aggregation over a $90 \mathrm{~min}$ period following addition of $500 \mathrm{pM}$ of 24base target DNA to a solution of $10 \mathrm{pM} \mathrm{P1+10} \mathrm{pM} \mathrm{P2} \mathrm{in} 3 \%$ dextran sulphate/0.3 M PBS buffer, comparing head-to-tail (H-T) and tail-to-tail (T-T) probe combinations for different lengths of probe DNA.

Table 1. Comparison of melting temperature values for a range of different probe combinations when 10 pM P1 + 10 pM P2 mixed with 5 nM Target ${ }_{144}$ in $3 \%$ dextran sulphate/0.3 M PBS buffer. Note: temperature could not be increased above $90{ }^{\circ} \mathrm{C}$, hence unable to get exact $\mathrm{T}_{\mathrm{m}}$ for final probe combination.

\begin{tabular}{cccc}
\hline Probes & Orientation & Duplex Length (bp) & $\left.\mathbf{T}_{\mathbf{m}} \mathbf{(}^{\circ} \mathbf{C}\right)$ \\
\hline $\mathrm{P} 1_{12}+\mathrm{P} 2_{12}$ & Head-Tail & 24 & $50.2 \pm 0.6$ \\
$\mathrm{P} 1_{18}+\mathrm{P} 2_{12}$ & Head-Tail & 30 & $59.5 \pm 0.4$ \\
$\mathrm{P} 1_{24}+\mathrm{P} 2_{12}$ & Head-Tail & 36 & $63.9 \pm 0.5$ \\
$\mathrm{P} 1_{12}+\mathrm{P} 2_{12}$ & Tail-Tail & 24 & $58.2 \pm 0.1$ \\
$\mathrm{P} 1_{18}+\mathrm{P} 2_{12}$ & Tail-Tail & 30 & $65.9 \pm 0.5$ \\
$\mathrm{P} 1_{24}+\mathrm{P} 2_{12}$ & Tail-Tail & 36 & $71.1 \pm 1.7$ \\
$\mathrm{P} 1_{18}+\mathrm{P} 2_{18}$ & Tail-Tail & 36 & $72.7 \pm 0.1$ \\
$\mathrm{P} 1_{24}+\mathrm{P} 2_{18}$ & Tail-Tail & 42 & $83.7 \pm 0.2$ \\
$\mathrm{P} 1_{24}+\mathrm{P} 2_{24}$ & Tail-Tail & 48 & $88.1 \pm 0.3$ \\
$\mathrm{P} 1_{30}+\mathrm{P} 2_{30}$ & Tail-Tail & 60 & $>88$ \\
\hline
\end{tabular}

